\title{
Clinical and biochemical characteristics of infants with prolonged neonatal jaundice
}

\author{
Sylvia LY Siu *, Lilian WM Chan, Albert NS Kwong
}

\section{A B S T R A C T}

Introduction: Protocols for investigating neonatal prolonged jaundice vary and the yield from screening has not been assessed. International guidelines recommend establishing cholestasis before proceeding to investigate the underlying pathology. However, in most hospitals administered by the Hospital Authority, full liver function is checked at the first neonatal jaundice clinic visit. To study the diagnostic yield of this approach, we carried out a retrospective study of all infants referred for prolonged jaundice.

Methods: Attendance records from the neonatal jaundice clinic at the Tuen Mun Hospital, Hong Kong, the clinical management system, and electronic patient records were used to retrieve epidemiological, clinical, and laboratory data, and patients' clinical progress.

Results: During the 8-month study period from 8 July 2015 to 8 March 2016, 1164 infants were referred to the neonatal jaundice clinic for prolonged jaundice. Among them, 16 (1.4\%) had conjugated hyperbilirubinaemia. Diagnoses included biliary atresia $(\mathrm{n}=1)$, cytomegalovirus (CMV) infection transient cholestasis $(\mathrm{n}=10)$. In total, $98(8.4 \%)$ infants had elevated alanine transaminase levels. Diagnoses included biliary atresia $(n=1)$, hepatic congestion related to congestive heart failure $(n=1)$, CMV infection $(n=5)$, neonatal hepatitis syndrome $(n=16)$, and non-specific elevated alanine transaminase $(n=75)$. In total, 59 infants had elevated alkaline phosphatase levels.

Conclusions: A stepwise approach is recommended, in which full liver function is checked and the underlying cause of jaundice is investigated only after confirming cholestasis.

\section{Hong Kong Med J 2018;24:270-6 DOI: 10.12809/hkmj176990}

${ }^{1}$ SLY Siu *, MB, ChB, FHKAM (Paediatrics)

${ }^{2}$ LWM Chan, MB, BS, FHKAM (Paediatrics)

${ }^{1}$ ANS Kwong, MB, BS, FHKAM (Paediatrics)

Department of Paediatrics and Adolescent Medicine, Tuen Mun Hospital, Tuen Mun, Hong Kong

${ }^{2}$ Ha Kwai Chung Child Assessment Centre, Kwai Chung, Hong Kong

* Corresponding author: siulys@ha.org.hk

New knowledge added by this study

- Among healthy infants with physiological or breastmilk jaundice, a transitional stage of cholestasis may occur when the jaundice is resolving. The lower conjugated bilirubin level and the downtrend of total bilirubin help to differentiate these infants from those with pathological cholestasis.

- Breastfed infants usually have mild elevation of alanine transaminase which may reflect higher metabolism instead of pathology. Therefore, checking alanine transaminase levels at the first neonatal jaundice clinic visit is not recommended because of the potential detrimental effect on breastfeeding rates.

- Late preterm infants with prolonged jaundice are at risk for osteopenia of prematurity.

Implications for clinical practice or policy

- A stepwise approach is recommended, checking full liver function and investigating the underlying cause only after confirming cholestasis.

- Bone profile blood test (alkaline phosphatase, albumin, calcium, and phosphate) is recommended for late preterm or low birth weight infants to screen for osteopenia of prematurity at the first neonatal jaundice clinic visit.

\section{Introduction}

All newborns have elevated unconjugated bilirubin concentrations relative to normal adult values. Two thirds or more of breastfed infants have unconjugated hyperbilirubinaemia that extends into the second and third weeks of life, and often up to age 8 to 12 weeks. ${ }^{1}$ Guidelines on the evaluation of cholestasis in infants recommend establishing cholestasis before proceeding to investigate the underlying pathology ${ }^{2,3}$ However, of the hospitals administered by the Hong Kong Hospital Authority that care for newborns, most check full liver function at the first Neonatal Jaundice Clinic (NNJC) visit.

The Food and Health Bureau established the Committee on Promotion of Breastfeeding in Hong Kong in 2014. Since then, there has been a substantial 
improvement in exclusive breastfeeding rates and a continuous upward trend in ever-breastfeeding rates. ${ }^{4}$

The Tuen Mun Hospital is a regional hospital in the New Territories West region of Hong Kong. An outpatient Neonatal Jaundice Clinic is available every Monday to Friday from 14:00 to 17:00 to treat infants with jaundice. The increasing breastfeeding rates have led to increasing numbers of infants with prolonged jaundice being referred to the NNJC.

The present study aimed to learn about the clinical and biochemical characteristics of prolonged neonatal jaundice, and to study the diagnostic yield of a full liver function check at first NNJC visit. We reviewed the clinical and laboratory records of infants visiting the NNJC between 8 July 2015 and 8 March 2016. On the basis of the findings, we propose a more effective evaluation procedure for prolonged neonatal jaundice.

\section{Methods}

Attendance records maintained by the NNJC included the registration number, sex, and attendance date of the patients. Using the recorded patient registration numbers, gestational age, birth weight, glucose6-phosphate dehydrogenase (G6PD) deficiency status, mode of feeding, phototherapy history, liver function test results, and clinical progress were retrieved from the clinical management system and electronic patient record. The study period was chosen for convenience. All infants who visited the NNJC during the study period were included to prevent possible selection bias. All suspicious data were verified by revisiting the electronic patient record to ensure data accuracy.

For gestational age, completed weeks of gestation were recorded. Preterm births were categorised as very preterm (before 34 weeks of gestation) or late preterm (between 34 weeks and 36 weeks 6 days of gestation). Full-term births were those at 37 weeks of gestation or later. Birth weights were categorised as very low $(<1500 \mathrm{~g})$, low (1500$2499 \mathrm{~g})$, normal (2500-3999 g), or high (>3999 g). Modes of feeding were categorised as exclusive breastfeeding, mixed feeding, or exclusive formula feeding.

\section{Data analysis}

The SPSS for Windows version 15.0 (SPSS Inc, Chicago [IL], United States) was used for all statistical analyses. Student's $t$ test and univariate analysis of variance (ANOVA) were used in comparing the outcomes between groups. Tukey's test was used for pairwise comparisons in ANOVA. Cross-tabulation was used to measure associations between binary outcome variables and binary predictor variables.

\section{長時間新生兒黃疸的臨床和生化特點研究 蕭鑾儀、陳慧雯、䣝毅山}

引言：長時間新生兒黃疸調查的協議各有不同，而篩查成效還未作評 估。國際準則建議檢視膽汁淤積的病理前應先作出診斷。然而, 大部 分醫院管理局轄下醫院會於首次在新生嬰兒黃疸診所就診時檢驗整套 肝功能指數。我們進行回顧性研究, 檢視這種方法的成效。

方法：本研究收集2015年7月 8 日至2016年3月 8 日期間轉介至新生嬰 兒黃㾝診所的新生兒的電腦記錄檔案, 從中獲得流行病學、臨床和生 化數據以及臨床進展數據。

結果：在研究的 8 個月內共納入 1164 名曾獲轉介至新生嬰兒黃疸診 所的新生兒。其中 16 名 $(1.4 \%)$ 新生兒有膽汁淤積並診斷為膽道 閉鎖 $(n=1)$ 、巨細胞病毒感染 $(n=3)$ 、新生兒肝炎綜合徵 $(n=2)$ 及短暫膽汁淤積 $(n=10)$ 。 98 名 $(8.4 \%)$ 新生兒有肝酶（丙氨酸 轉氨酶）上升；當中診斷包括膽道閉鎖 $(n=1)$ 、充血性心力衰竭 導致肝充血 $(n=1)$ 、巨細胞病毒感染 $(n=5)$ 、新生兒肝炎綜合徵 $(n=16)$ 及非特定肝酶（丙氨酸轉氨酶）上升（ $n=75 ） 。 59$ 名新生 兒有虞性磷酸酶上升。

結論：我們推薦逐步籂查的方法, 先行診斷膽汁淤積, 然後才檢驗整 套肝功能指數。

\section{Results}

\section{Demographic characteristics}

In total, 1164 infants (663 males, 501 females; maleto-female ratio $=1.3: 1$ ) with prolonged jaundice were referred to the NNJC during the 8-month study period. The gestational ages of the infants ranged from 29 to 41 weeks, and there were eight (0.69\%) very preterm infants and $94(8.08 \%)$ late preterm infants. The birth weights of the infants ranged from $1425 \mathrm{~g}$ to $4670 \mathrm{~g}$. The sample included only one $(0.09 \%)$ very low birth weight infant, in addition to 80 (6.87\%) low birth weight infants and 13 (1.12\%) high birth weight infants. In total, $34(5.13 \%)$ male infants and two $(0.40 \%)$ female infants had G6PD deficiency. The mode of feeding was exclusive breastfeeding in 648 (55.70\%) infants, mixed feeding in 400 (34.36\%) infants, and exclusive formula feeding in 114 (9.79\%) infants; the mode of feeding was not recorded in two infants. Among the 114 exclusively formula fed infants, $24(21.10 \%)$ had a history of breastfeeding. At the first NNJC visit, 70 (6.01\%) infants were 2 weeks old, 156 (13.40\%) 3 weeks old, 758 (65.12\%) 4 weeks old, 165 (14.18\%) 5 weeks old, 10 (0.86\%) 6 weeks old, and three (0.26\%) 7 weeks old. One $(0.09 \%)$ infant first visited at 8.7 weeks old and another $(0.09 \%)$ first visited at 11.4 weeks old.

\section{Full liver function test}

At the first NNJC visit, 1139 (97.90\%) infants received a full liver function test, which included taking measurements of alkaline phosphatase (ALP), calcium, and phosphate. In seven infants, 
low transcutaneous bilirubinometer readings were observed (peak reading, 42-86 $\mu \mathrm{mol} / \mathrm{L}$ ). Therefore, blood tests were not performed in these infants. At the first NNJC visit, 17 infants were seen by a doctor who adopted the stepwise approach and who screened only for cholestasis; one infant visiting on day 14 had only total bilirubin (TB) checked.

\section{Influence of breastfeeding on liver biochemistry}

There was a significant effect of mode of feeding on TB $\left(F_{2,1155}=18.058 ; \mathrm{P}<0.01\right)$. The mean $(\mathrm{M})$ TB levels in the exclusive breastfeeding group $(M=146.73)$ were significantly higher than those in the mixed feeding group $(M=131.05)$ and in the exclusive formula feeding group $(M=121.21)$. Tukey's test showed significant differences in TB levels between the exclusive breastfeeding and the mixed feeding groups $(\mathrm{P}<0.01)$ and between the exclusive breastfeeding and the exclusive formula feeding groups $(P<0.01)$. There was no significant difference in TB level between the mixed feeding and exclusive formula feeding groups.

There was a significant effect of feeding on alanine transaminase (ALT) levels $\left(F_{2,1133}=15.015\right.$; $\mathrm{P}<0.01)$. The ALT levels in the exclusive breastfeeding group $(\mathrm{M}=21.79)$ were significantly higher than those in the mixed feeding group $(M=18.97)$ and in the exclusive formula feeding group $(\mathrm{M}=16.55)$.
Tukey's test showed significant differences in ALT levels between the exclusive breastfeeding and the mixed feeding groups $(\mathrm{P}<0.01)$ and between the exclusive breastfeeding and the exclusive formula feeding groups $(\mathrm{P}<0.01)$. There was no significant difference in ALT levels between the mixed feeding and exclusive formula feeding groups.

There was a significant effect of feeding on ALP $\left(F_{2,1135}=6.276 ; \mathrm{P}<0.01\right)$. The ALP levels in the exclusive breastfeeding group $(\mathrm{M}=348.83)$ were significantly higher than those in the mixed feeding group $(M=329.08)$ and in the exclusive formula feeding group $(M=327.76)$. Tukey's test showed a significant difference in ALP level between the exclusive breastfeeding and the mixed feeding groups $(\mathrm{P}<0.01)$. There was no significant difference in ALP levels between the exclusive breastfeeding and the exclusive formula feeding groups $(\mathrm{P}=0.07)$ or between the mixed feeding and the exclusive formula feeding groups $(\mathrm{P}=0.99)$.

There was a significant effect for infants who had received phototherapy $(t(1141)=3.57 ; \mathrm{P}<0.01)$. Infants who had received phototherapy had higher TB levels $(M=147.12)$ than those who had not $(M=135.06)$.

There was no significant difference in TB levels between male and female infants. There was also no statistically significant difference in TB levels between G6PD-deficient and G6PD-sufficient infants.

TABLE I. Demographic and laboratory characteristics of cholestatic infants

\begin{tabular}{|c|c|c|c|c|c|c|c|c|c|c|c|}
\hline $\begin{array}{l}\text { Case } \\
\text { No. }\end{array}$ & Diagnosis & GA, wk & BW, kg & Sex & Day & Milk & $\begin{array}{c}\text { G6PD } \\
\text { deficient }\end{array}$ & $\begin{array}{c}\text { TB, } \\
\mu \mathrm{mol} / \mathrm{L}\end{array}$ & $\begin{array}{c}\mathrm{DB}, \\
\mathrm{mmol} / \mathrm{L}\end{array}$ & ALP, U/L & ALT, U/L \\
\hline 1 & Biliary atresia & 41 & 2.82 & $\mathrm{~F}$ & 35 & $\mathrm{AF}$ & No & 123 & 92 & 576 & 64 \\
\hline 2 & Cytomegalovirus & 38 & 2.94 & M & 34 & Mix & No & 116 & 47 & 439 & 24 \\
\hline 3 & Cytomegalovirus & 39 & 3.59 & M & 29 & EBF & No & 66 & 39 & 586 & 21 \\
\hline 4 & Cytomegalovirus & 38 & 3.04 & M & 34 & EBF & No & 93 & 22 & 380 & 39 \\
\hline 5 & $\begin{array}{l}\text { Neonatal hepatitis } \\
\text { syndrome }\end{array}$ & 35 & 2.57 & M & 33 & EBF & No & 90 & 64 & 624 & 58 \\
\hline 6 & $\begin{array}{l}\text { Neonatal hepatitis } \\
\text { syndrome }\end{array}$ & 35 & 2.73 & M & 33 & EBF & No & 110 & 76 & 611 & 83 \\
\hline 7 & Transient cholestasis & 38 & 3.17 & M & 37 & EBF & No & 78 & 18 & 595 & 23 \\
\hline 8 & Transient cholestasis & 39 & 3.18 & M & 34 & EBF & No & 84 & 20 & 444 & 9 \\
\hline 9 & Transient cholestasis & 39 & 4.09 & M & 32 & $\mathrm{AF}$ & No & 100 & 23 & 381 & 19 \\
\hline 10 & Transient cholestasis & 38 & 3.51 & $\mathrm{~F}$ & 38 & EBF & No & 58 & 25 & 336 & 48 \\
\hline 11 & Transient cholestasis & 40 & 3.40 & M & 32 & AF & No & 62 & 27 & 376 & 18 \\
\hline 12 & Transient cholestasis & 40 & 3.55 & M & 28 & Mix & Yes & 124 & 33 & 404 & 20 \\
\hline 13 & Transient cholestasis & 40 & 2.97 & M & 34 & Mix & No & 139 & 51 & 412 & 41 \\
\hline 14 & Transient cholestasis & 38 & 2.60 & $\mathrm{~F}$ & 33 & Mix & No & 87 & 18 & 629 & 19 \\
\hline 15 & Transient cholestasis & 39 & 3.29 & M & 31 & Mix & No & 121 & 25 & 617 & 27 \\
\hline 16 & Transient cholestasis & 38 & 3.36 & M & 40 & EBF & No & 98 & 60 & 405 & 29 \\
\hline
\end{tabular}

Abbreviations: $\mathrm{AF}=$ artificial formula; $\mathrm{ALP}=$ alkaline phosphatase; $\mathrm{ALT}=$ alanine transaminase; $\mathrm{BW}=$ birth weight; $\mathrm{DB}=$ direct bilirubin; $\mathrm{EBF}=$ exclusive breastfeeding; $F$ = female; $G 6 P D=$ glucose-6-phosphate dehydrogenase; $G A=$ gestational age; $M$ = male; Mix = mixed feeding; $T B=$ total bilirubin 


\section{Cholestasis}

At the first NNJC visit, 16 (1.4\%) infants had conjugated hyperbilirubinaemia. Diagnoses included biliary atresia in one, cytomegalovirus (CMV) infection in three, neonatal hepatitis syndrome in two, and transient cholestasis in 10 infants (Table 1). All infants were thriving well and did not have dark urine or pale stool at their first NNJC visit.

\section{Elevated alanine transaminase level}

At the first NNJC visit, 98 (8.4\%) infants had elevated ALT levels. The reference range used for ALT level is $5 \mathrm{U} / \mathrm{L}$ to $33 \mathrm{U} / \mathrm{L}$. The proportion of infants that were followed up increased with increasing ALT level, as shown in Figure 1. Specific causes for elevated ALT level included biliary atresia in one infant, hepatic congestion related to congestive heart failure in one infant, and CMV in five infants (Table 2). Of the remaining 91 infants with elevated ALT levels, 16 had neonatal hepatitis syndrome and 75 had nonspecific elevated ALT levels.

Urine tests revealed CMV infection in five infants with elevated ALT levels; CMV infection was believed to be acquired postnatally. These infants were all full-term births of normal birth weight and were asymptomatic. Of these five infants, four were exclusively breastfed and one was fed a mix of breastmilk and formula. After their elevated ALT levels resolved, three infants were discharged from the NNJC and the other two were followed up for coincidental findings (developmental concern in one and familial small head in the other) and not for concern over CMV infection. These five infants and the 75 infants with non-specific elevated ALT were all healthy and asymptomatic and had good weight gain; their elevated ALT levels resolved without treatment.

In this study, inflammation of the liver occurring in early infancy that could not be attributed to a specific cause of liver disease was termed neonatal hepatitis syndrome. The peak ALT levels and the duration of elevated ALT levels in 16 infants with neonatal hepatitis syndrome are shown in Figure 2. The duration of elevated ALT level ranged from 13 to 69 weeks and was shorter than 28 weeks in only four infants. Infants with neonatal hepatitis syndrome were followed up until ALT

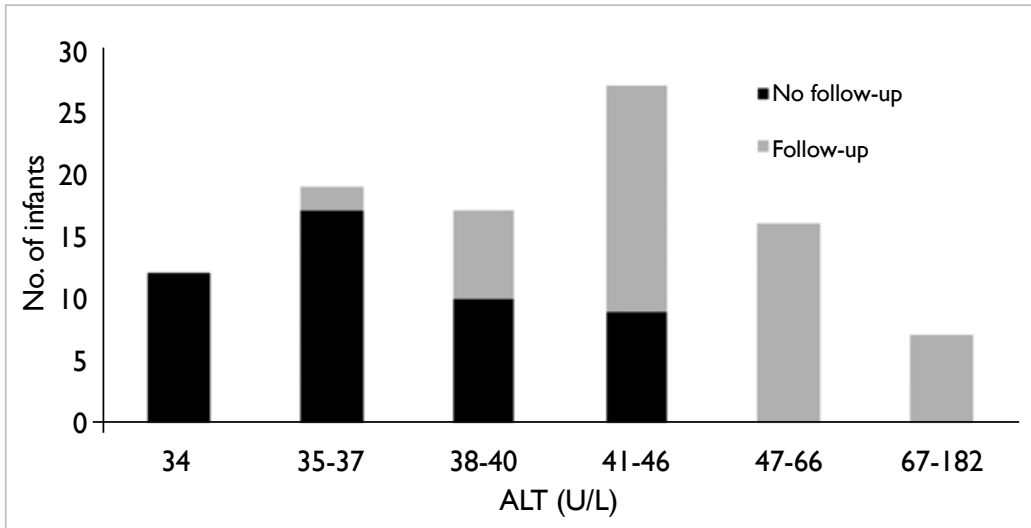

FIG I. Infants with elevated ALT at the first visit Abbreviation: $\mathrm{ALT}=$ alanine transaminase

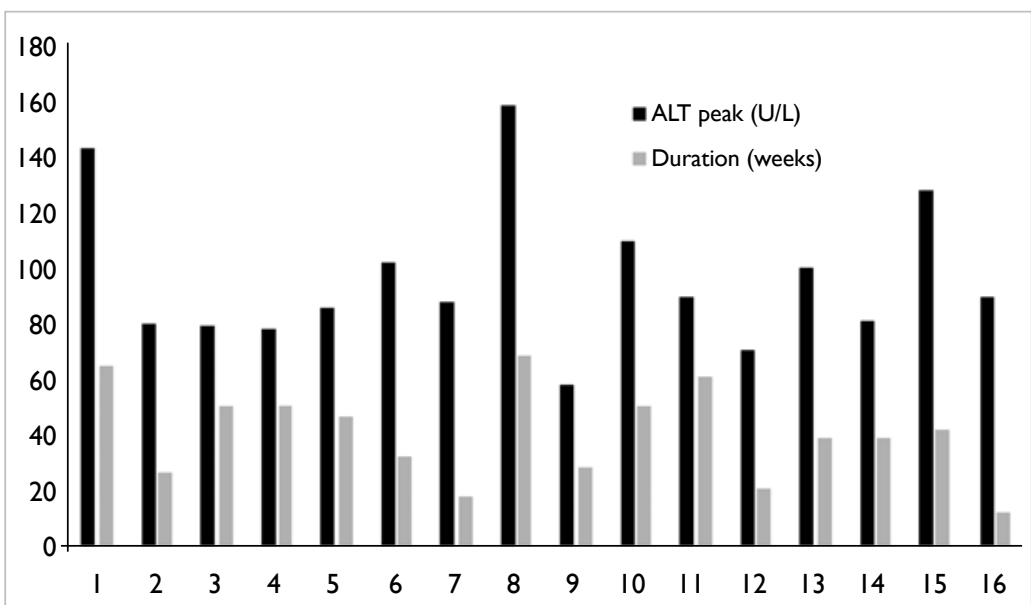

FIG 2. Peak and duration of elevated ALT levels in 16 infants with neonatal hepatitis syndrome

Abbreviation: $\mathrm{ALT}=$ alanine transaminase

TABLE 2. Specific pathology of elevated alanine transaminase level

\begin{tabular}{lcc}
\hline Pathology & Peak ALT level, U/L & Duration of elevated ALT level \\
\hline Biliary atresia & 91 (Before Kasai operation) & Indefinite \\
$\begin{array}{l}\text { Congestive heart failure (atrial septal defect and } \\
\text { ventricular septal defect) }\end{array}$ & 51 & 4 Months \\
Cytomegalovirus & 58 & 4 Months \\
Cytomegalovirus & 104 & 7 Months \\
Cytomegalovirus & 128 & 12 Months \\
Cytomegalovirus & 201 & 10 Months \\
Cytomegalovirus & 60 & 11 Months \\
\hline
\end{tabular}

Abbreviation: $\mathrm{ALT}=$ alanine transaminase 
levels returned to normal and then for an average of 1 month longer.

In 75 infants who were otherwise healthy, non-specific elevated ALT levels were within double the usual upper limit. These infants were either not followed up or their liver function was monitored periodically (time intervals in months determined on case-by-case basis) with limited diagnostic testing.

\section{Elevated alkaline phosphatase level and low phosphate level}

The mode of feeding, gestational age, and birth weight were all found to affect ALP levels. Among 132 preterm or low birth weight infants, 21 (15.9\%) had elevated ALP, compared with 31 (3.0\%) among 1032 full-term and normal birth weight infants. For preterm or low birth weight infants versus term infants with birth weight $>2499 \mathrm{~g}$, the odds ratio for elevated ALP was 6.109 (95\% confidence interval=3.394-10.994) [Table 3]. Among 1139infants who underwent full liver function tests, 10 had low phosphate levels: seven had concomitant high ALP and low phosphate levels, and the remaining two late preterm infants and one full-term infant had isolated low phosphate levels.

\section{Discussion}

We found that TB levels were significantly higher in the exclusive breastfeeding and mixed feeding groups than in the exclusive formula feeding group. Eight infants that were exposed to breastmilk and two exclusively formula-fed infants had transient cholestasis. Our findings also revealed that ALT levels were significantly higher in the exclusive breastfeeding and mixed feeding groups than in the exclusive formula feeding group. Besides, preterm or low birth weight infants had increased odds of high ALP levels compared with term infants with birth weight $>2499$ g.

\section{Cholestasis}

Breast milk jaundice (BMJ) was first described by Newman and Gross in 1963..$^{5}$ Subsequently, BMJ has been reported to be associated with increased conjugated and unconjugated bilirubin levels. ${ }^{6}$ In
1991, investigators in Japan studied 58 breastfed infants with indirect hyperbilirubinaemia and found that $18(31 \%)$ with BMJ had elevated ALP, gamma-glutamyltranspeptidase or serum bile acid, reflecting alterations in the hepatobiliary system. ${ }^{7}$ The serum bile acid levels in patients with BMJ are similar to those with cholestatic jaundice caused by diseases such as extrahepatic biliary atresia. ${ }^{7}$ This finding suggests that BMJ may be caused by hepatic dysfunction related to cholestasis. Moreover, for infants with BMJ and increased fasting serum bile acid levels, discontinuation of breastfeeding did not cause a rapid normalisation of the serum TB levels. ${ }^{7}$ This observation suggests that hyperbilirubinaemia in infants with increased serum bile acid levels is not directly related to breastfeeding. Our finding of transient cholestasis in $1.75 \%(2 / 114)$ of exclusive formula feeding infants versus $0.76 \% \quad(8 / 1048)$ exclusive breast milk feeding and mixed feeding infants supported the hypothesis that cholestasis is not related to breastfeeding.

In this study, we used the term 'transient cholestasis' to describe the delay in the decline of conjugated bilirubin levels, as observed in 10 infants. Because TB levels declined first without a concomitant decline or even with an increase in conjugated bilirubin levels, there seemed to be a transitional stage of cholestasis. When TB levels decreased further, the conjugated bilirubin levels then decreased. Moreover, the conjugated bilirubin levels in these 10 infants were lower than those with biliary atresia or hepatitis. This finding supports the use of direct bilirubin as a surrogate marker in assessing the severity of cholestasis to ensure optimal timing of hepatobiliary scanning. ${ }^{8}$

\section{Elevated alanine transaminase level}

In 1981, Landaas et $\mathrm{al}^{9}$ first reported a significant difference in ALT levels between breastfed and formula-fed infants. They proposed a normal range for ALT level of 14 to 84 IU/L until 4.5 months of age. In 1984, Gómez et $\mathrm{al}^{10}$ examined 2099 outpatient children and found that ALT levels (40-97 IU/L, 3rd to 97 th percentile) were higher in children $<1$ year than in older children. In 2003, investigators in Denmark found higher mean serum bilirubin,

TABLE 3. Infants with isolated elevated alkaline phosphatase and low phosphate

\begin{tabular}{|c|c|c|}
\hline & Total No. & No. (\%) with elevated ALP \\
\hline Infants who received full liver function test & 1139 & $52(4.6)$ \\
\hline Preterm infants or birth weight $<2.5 \mathrm{~kg}^{\star}$ & 132 & $21(15.9) \dagger$ \\
\hline Full-term infants or birth weight $\geq 2.5 \mathrm{~kg}$ & 1032 & $31(3.0) \ddagger$ \\
\hline $\begin{array}{l}\text { Abbreviation: ALP = alkaline phosphatase } \\
* \text { Odds ratio of elevated ALP level=6.109 }(959 \\
\dagger \text { Six infants had concomitant low phosphate } \\
\ddagger \text { One infant had concomitant low phosphate }\end{array}$ & $394-10.994)$ & \\
\hline
\end{tabular}


albumin, and aspartate transaminase (AST) levels in healthy exclusively breastfed full-term infants; they also found a strong positive association between AST and insulin-like growth factor-1 levels at 2 months $(r=0.47 ; \mathrm{P}=0.004) .{ }^{11}$ Protein levels in breast milk are lower than those in infant formulas. Serum albumin levels have been used to evaluate the adequacy of protein levels in infant formula. Thus, the finding of higher serum albumin levels in breastfed infants than formula feeding infants suggests that there were no protein deficiencies or abnormalities affecting albumin production in breastfed infants. ${ }^{11}$ Insulin-like growth factor- 1 is an anabolic hormone in infants; thus, those authors believed that the most likely explanation for the elevated AST values among breastfed infants is a stimulation of liver metabolism through one of several growth factors in human milk. ${ }^{11}$ Therefore, the higher AST levels in breastfed infants were believed to be a reflection of a higher liver metabolism, rather than a reflection of liver cell damage. Alanine transaminase is present primarily in the liver and thus is a more specific marker of hepatocellular cell injury. Aspartate transaminase is present in the liver and other organs, a less specific marker of hepatocellular function. The aforementioned study used AST in a restrictive sense to reflect liver biochemistry. Therefore, the higher ALT levels among breastfed infants should logically be interpreted by the same token as reflection of higher liver metabolism rather than liver cell injury.

In the present study, other than those in which $\mathrm{CMV}$ was identified, findings were negative. Elevated ALT concentrations resolved in all infants other than the infant with biliary atresia. These findings support the hypothesis that elevated ALT in breastfed infants is a reflection of higher metabolism rather than of any pathology.

\section{Isolated elevated alkaline phosphatase level and low phosphate level}

Serum ALP is derived predominately from the liver and bones. In the present study, elevated ALP in seven infants was of hepatic origin. Tests of ALP's heat stability index revealed that 52 infants with isolated ALP elevation were of bone origin.

In this study, 21 (15.9\%) preterm or low birth weight infants had isolated elevated ALP (Table 3). Among the eight very preterm infants, only one had isolated elevated ALP and none had low phosphate. Among 94 late preterm infants, 20 (21.3\%) had isolated elevated ALP and among these, six (30\%) also had low phosphate.

The biochemical characteristics of high ALP and low phosphate are compatible with osteopenia of prematurity. During pregnancy, calcium and phosphorus are actively transferred from the mother to the fetus, reaching a peak accretion rate at 32 to 36 weeks of gestation. The third trimester is the period of most active growth and the increased accretion rate is in response to the higher fetal needs for the developing skeleton. As a result, in preterm infants, calcium and phosphate requirements increase with decreasing gestational age, to compensate for the loss of accretion of these minerals. In the present study, very preterm infants were managed in neonatal wards for long durations; therefore, this need for increased mineral supplements was recognised and addressed. However, late preterm infants may not be clearly distinguished from full-term infants, and their need for additional minerals may not be apparent or addressed during their short hospital stay.

\section{Diagnostic yield of full liver function test at first neonatal jaundice clinic visit}

Breastfed infants have been reported to acquire CMV via breastmilk. ${ }^{12,13}$ Cytomegalovirus excreted in breastmilk is likely caused by reactivated infection in the presence of maternal antibody transferred transplacentally. ${ }^{12}$ This type of milk-borne CMV infection apparently protects children from CMV diseases and the seropositivity for CMV may protect the next generation from CMV inclusion disease.

Poddighe et $\mathrm{al}^{14}$ reported a full-term breastfed infant with prolonged jaundice who had undergone extensive tests but with negative findings. Liver function test results returned to normal by age 7 months when breastmilk intake was significantly reduced. The authors ${ }^{14}$ then proposed that, in otherwise healthy infants and in the absence of risk factors, elevated ALT levels should be monitored for 7 months before performing further sophisticated tests. If this proposal were applied to the present study, only four infants had elevated ALT for less than 7 months and would have avoided further tests.

\section{Proposed prolonged neonatal jaundice evaluation}

The above discussion suggests that measuring ALT levels at the first NNJC visit is of limited benefit. For jaundiced infants, repeated follow-up examinations for elevated ALT levels may increase the risk of premature cessation of breastfeeding and of development of vulnerable child syndrome. ${ }^{15}$ Therefore, we propose measuring ALT levels only after noting cholestasis.

In the present study, late preterm infants were identified to be at high risk of having ALP elevation. Preterm or low birth weight infants accounted for $<10 \%$ of those visiting the NNJC. In addition to cholestasis screening at the first NNJC visit, ALP, albumin, calcium, and phosphate should be checked for late preterm or low birth weight infants.

Vitamin D deficiency has been reported in 18\% of Hong Kong women. ${ }^{16}$ In this study, the prevalence of elevated ALP was $4.6 \%$. The recently published global consensus on prevention and management 
of nutritional rickets recommends vitamin D supplementation at 400 IU daily in all infants, independent of their mode of feeding in the first year of life. ${ }^{17}$ Therefore, the best way to protect infants may be to educate pregnant mothers to take vitamin D supplements during pregnancy and to give $400 \mathrm{IU}$ daily vitamin D supplements to their infants. ${ }^{17}$

\section{Limitations}

Information bias and selection bias are two potential limitations of our study. First, for simplicity, all infants taking just one mouthful of breastmilk and those taking just one mouthful of formula were classified in the mixed feeding group. In the mixed feeding group, this information bias may mask the effect of breastfeeding on liver biochemistry. Second, the study period was chosen for convenience and not at random. This may create selection bias because outbreaks of diseases in infants tend to create clusters of clinic visits within a certain timeframe.

\section{Conclusions}

For full-term and normal or high birth weight infants, the most effective way to manage prolonged neonatal jaundice is to screen for cholestasis before full liver function examination. For late preterm or low birth weight infants, the most effective way to manage prolonged neonatal jaundice is to screen for cholestasis and to check bone profile (ALP, albumin, calcium, and phosphate) at the first NNJC visit.

\section{Author contributions}

All authors have made substantial contributions to the concept or design of this study; acquisition of data; analysis or interpretation of data; drafting of the article; and critical revision for important intellectual content.

\section{Acknowledgement}

The authors thank Ms CK Ho and Mr WF Wu for maintaining the neonatal jaundice clinic attendance records that made this retrospective study possible.

\section{Funding/support}

This research received no specific grant from any funding agency in the public, commercial, or not-for-profit sectors.

\section{Declaration}

All authors have disclosed no conflicts of interest. All authors had full access to the data, contributed to the study, approved the final version for publication, and take responsibility for its accuracy and integrity. This paper was presented orally in Joint Annual Research \& Scientific Meeting 2017, 19 August 2017, Hong Kong.

\section{Ethical approval}

Ethical approval for the study was obtained and patient/ parental consent was waived by the New Territories West Cluster Clinical and Research Ethics Committee.

\section{References}

1. Academy of Breastfeeding Medicine Protocol Committee. ABM clinical protocol \#22: guidelines for management of jaundice in the breastfeeding infant equal to or greater than 35 weeks' gestation. Breastfeed Med 2010;5:8793.

2. Fawaz R, Baumann U, Ekong U, et al. Guideline for the evaluation of cholestatic jaundice in infants: joint recommendations of the North American Society for Pediatric Gastroenterology, Hepatology, and Nutrition and the European Society for Pediatric Gastroenterology, Hepatology, and Nutrition. J Pediatr Gastroenterol Nutr 2017;64:154-68.

3. Moyer V, Freese DK, Whitington PF, et al. Guideline for the evaluation of cholestatic jaundice in infants: recommendations of the North American Society for Pediatric Gastroenterology, Hepatology and Nutrition. J Pediatr Gastroenterol Nutr 2004;39:115-28.

4. Family Health Service, Department of Health, HKSAR Government. Breastfeeding Survey 2017.

5. Newman AJ, Gross S. Hyperbilirubinemia in breast-fed infants. Pediatrics 1963;32:995-1001.

6. Winfield CR, Macfaul R. Clinical study of prolonged jaundice in breast- and bottle-fed babies. Arch Dis Child 1978;53:506-7.

7. Tazawa Y, Abukawa D, Watabe M, et al. Abnormal results of biochemical liver function tests in breast-fed infants with prolonged indirect hyperbilirubinaemia. Eur J Pediatr 1991;150:310-3.

8. Siu LY, Wong KN, Li KW, et al. Outcome of hepatobiliary scanning: preterm versus full-term cholestatic infants. J Paediatr Child Health 2013;49:E46-51.

9. Landaas S, Skrede S, Steen JA. The levels of serum enzymes, plasma proteins and lipids in normal infants and small children. J Clin Chem Clin Biochem 1981;19:107580.

10. Gómez P, Coca C, Vargas C, et al. Normal referenceintervals for 20 biochemical variables in healthy infants, children and adolescents. Clin Chem 1984;30:407-12.

11. Jørgensen $\mathrm{MH}$, Ott P, Juul A, et al. Does breast feeding influence liver biochemistry? J Pediatr Gastroenterol Nutr 2003;37:559-65.

12. Minamishima I, Ueda K, Minematsu T, et al. Role of breast milk in acquisition of cytomegalovirus infection. Microbiol Immunol 1994;38:549-52.

13. Hamprecht K, Maschmann J, Vochem M, et al. Epidemiology of transmission of cytomegalovirus from mother to preterm infants by breastfeeding. Lancet 2001;357:513-8.

14. Poddighe D, Castelli L, Marseglia GL, et al. Prolonged, but transient, elevation of liver and biliary functions tests in a healthy infant affected with breast milk jaundice. BMJ Case Rep 2014;pii:bcr2014204124.

15. Kemper K, Forsyth B, McCarthy P. Jaundice, terminating breast-feeding and the vulnerable child. Pediatrics 1989;84:773-8.

16. Woo J, Lam CW, Leung J, et al. Very high rates of vitamin $\mathrm{D}$ insufficiency in women of child-bearing age living in Beijing and Hong Kong. Br J Nutr 2008;99:1330-4.

17. Munns CF, Shaw N, Kiely M, et al. Global consensus recommendations on prevention and management of nutritional rickets. J Clin Endocrinol Metab 2016;10:394415. 